\title{
The influence of nociceptive and neuropathic pain states on the processing of acute electrical nociceptive stimulation: a dynamic causal modeling study.
}

Lisa Goudman ${ }^{1-3}$ PhD., Daniele Marinazzo ${ }^{4}$ PhD., Frederik Van de Steen ${ }^{4}$ PhD., Guy Nagels ${ }^{3,5-6} \mathrm{PhD}$., Ann De Smedt ${ }^{3,7}$ PhD., Eva Huysmans ${ }^{2,8-12}$ PhD., Koen Putman ${ }^{8-9}$ PhD., Ronald Buyl ${ }^{13}$ PhD., Kelly Ickmans ${ }^{2,10-12} \mathrm{PhD}$. , Jo Nijs ${ }^{2,10-11} \mathrm{PhD}$., Iris Coppieters ${ }^{2,10-11} \mathrm{PhD}$., Maarten Moens ${ }^{1,3,14} \mathrm{PhD}$.

1. Department of Neurosurgery, Universitair Ziekenhuis Brussel, Laarbeeklaan 101, 1090 Brussels, Belgium

2. Pain in Motion international research group, www.paininmotion.be

3. Center for Neurosciences (C4N), Vrije Universiteit Brussel (VUB), Laarbeeklaan 103, 1090 Brussels, Belgium

4. Department of Data Analysis, University of Ghent, 9000 Ghent, Belgium.

5. National MS Center, Neurology, Vanheylenstraat 16, 1820 Melsbroek, Belgium.

6. St Edmund Hall, Queen's Lane, Oxford OX1 4AR, United Kingdom

7. Department of Physical Medicine and Rehabilitation, Universitair ziekenhuis Brussel, Laarbeeklaan 101, 1090 Brussels, Belgium

8. Department of Public Health (GEWE), Faculty of Medicine and Pharmacy, Vrije Universiteit Brussel, Laarbeeklaan 103, 1090 Brussels, Belgium.

9. Interuniversity Centre for Health Economics Research (I-CHER).

10. Department of Physical Medicine and Physiotherapy, Universitair Ziekenhuis Brussel, Laarbeeklaan 101, 1090 Brussels, Belgium.

11. Department of Physiotherapy, Human Physiology and Anatomy, Faculty of Physical Education \& Physiotherapy, Vrije Universiteit Brussel, Laarbeeklaan 103, 1090 Brussels, Belgium

12. Research Foundation - Flanders (FWO), Brussels

13. Department of Biostatistics and Medical Informatics, Faculty of Medicine and Pharmacy, Vrije Universiteit Brussel, Laarbeeklaan 103, 1090 Brussels, Belgium.

14. Department of Radiology, Universitair Ziekenhuis Brussel, Laarbeeklaan 101, 1090 Brussels, Belgium

Address of correspondence and reprints requests to Prof. Dr. Maarten Moens (MD, PhD), department of Neurosurgery, Universitair Ziekenhuis Brussel, Laarbeeklaan 101, 1090 Brussels, Belgium. E-mail: maarten.moens@uzbrussel.be, tel:+3224776012, fax: +32/2 4775570. 


\section{Abstract}

Introduction. Despite the worldwide increase in prevalence of chronic pain and the subsequent scientific interest, researchers studying the brain and brain mechanisms in pain patients have not yet clearly identified the exact underlying disease. Quantifying the neuronal interactions in electrophysiological data could help us gain insight into the complexity of chronic pain. Therefore, the aim of this study is to examine how different underlying pain states affect the processing of nociceptive information.

Methods. Twenty healthy participants, 20 patients with non-neuropathic low back-related leg pain and 20 patients with neuropathic failed back surgery syndrome received painful electrical stimulation at the right sural nerve with simultaneous electroencephalographic recordings. Dynamic Causal Modeling (DCM) was used to infer hidden neuronal states within a Bayesian framework.

Results. Pain intensity ratings and stimulus intensity of the nociceptive stimuli did not differ between groups. Compared to healthy participants, both patient groups had the same winning DCM model, with an additional forward and backward connection between the somatosensory cortex and right dorsolateral prefrontal cortex.

Discussion. The additional neuronal connection with the prefrontal cortex as seen in both pain patient groups could be a reflection of the higher attention towards pain in pain patients and might be explained by the higher levels of pain catastrophizing in these patients.

Conclusion. In contrast to the similar pain intensity ratings of an acute nociceptive electrical stimulus between pain patients and healthy participants, the brain is processing these stimuli in a different way.

Keywords: nociceptive stimulation, dynamic causal modeling, effective connectivity, electroencephalography 


\section{Introduction}

Chronic pain has a prevalence of 19.6\% in individuals between 20 and 59 years old (Meucci et al., 2015), with the lower back being one of the most common body locations for developing chronic pain (Macfarlane, 2016). This high prevalence has led to the recognition of low back pain (LBP) as a significant public health problem (Amorim et al., 2016). Despite the functional disability, impairment of psychological status and low quality of life related to chronic LBP, a well-defined targeted and effective treatment strategy for these patients is still lacking (Hong et al., 2014; Webster and Markman, 2014). The fact that pain is a very complex and multidimensional experience, with different psychological processes (e.g., pain catastrophizing) that are mediating the net pain response (Edwards et al., 2016; Meints and Edwards, 2018), is not helpful to find suitable treatments for all patients. Revealing the exact pathophysiological mechanisms underlying different types of pain would be of great value for pain treatment.

A Voxel-Based-Morphometry study emphasized the role of pain catastrophizing in patients with low back pain, which was significantly associated with cortical gray matter volume in brain areas involved in processing attentional, sensory and affective aspects of pain (Chehadi et al., 2017). Pain catastrophizing is a construct that comprises elements of rumination (i.e., an anxious preoccupation with pain and the inability to inhibit pain-related thoughts and fears), magnification (i.e., the tendency to amplify the significance of pain with respect to implications for one's global health), and helplessness (i.e., despair surrounding perceived inability to control one's pain experience) (Burns et al., 2015; Sullivan et al., 1995). Pain catastrophizing is an important predictor of functioning and disability among individuals with chronic pain (Craner et al., 2016). The presence of chronic LBP itself, is associated with several functional and structural anatomical alterations, such as for example increased cortical thickness of the dorsolateral prefrontal cortex (Apkarian, 2015; Seminowicz et al., 2011). A recent systematic review evaluating brain alterations and clinical measures, concluded that these brain alterations should be taken into account when treating patients with chronic musculoskeletal pain by utilizing a biopsychosocially-driven approach that is also addressing the central nervous system (Coppieters et al., 2016). This emphasizes the importance of evaluating how these alterations are influencing brain networks.

Previously, several differences between patients with chronic nociceptive and neuropathic pain were noted. Patients with predominantly neuropathic chronic low back pain report higher pain intensity scores, poorer physical and mental health related quality of life, greater signs of depression and psychological distress and increased sensory thresholds to tactile stimulation compared to patients with predominantly nociceptive pain (Spahr et al., 2017). Studies exploring brain alterations in patients with predominant nociceptive versus neuropathic chronic pain are rather scarce. Gustin et al. (2011) explored changes in regional brain anatomy in patients with non-neuropathic and neuropathic orofacial pain. Patients with neuropathic pain revealed significant changes in gray matter volume compared to non-neuropathic pain patients. More specifically, gray matter volume of neuropathic pain patients was reduced in the primary somatosensory cortex, anterior insular, putamen, nucleus accumbens, and the thalamus, whereas gray matter volume was increased in the posterior insula (Gustin et al., 2011). 
One way to further elucidate the underlying brain mechanisms is by exploring effective connectivity. Within this concept, researchers evaluate the influence that one neuronal system is exerting over another with a directional relationship (Friston, 1994). Dynamic Causal Modeling (DCM) is a type of connectivity analysis to infer hidden neuronal states behind measured brain responses, based on a Bayesian framework (Stephan et al., 2010). DCM models are generative models of the brain responses, which provide posterior parameter estimates including information about the strength of connections between neuronal populations and the context-dependent modulation (Stephan et al., 2010). This process was previously described as "performing a biological informed source reconstruction with the added constraint that the activity in one source has to be caused by activity in another, in a biologically plausible fashion" (David et al., 2006). In the present study, DCM will be used to further explore the interactions between brain regions in healthy participants, patients with nociceptive LBP (presurgical patients with nociceptive low back-related leg pain) and patients with neuropathic LBP due to Failed Back Surgery Syndrome. The interactions between brain regions will be evaluated when providing an acute nociceptive stimulus. Therefore, the aim of this study is to explore the neuronal architecture that might underlie acute nociceptive stimuli processing and how this is altered by nociceptive and neuropathic pain states. Additionally, after identifying the underlying model(s), inferences on parameter estimates will be made to observe differences in brain connections between healthy participants and patients with different pain profiles.

\section{Results}

\section{$\underline{2.1 \text { Pain thresholds }}$}

The median EDT and EPT were not significantly different between the three groups (EDT $\chi 2(2)=1.74$, $p=0.42$; EPT $\chi 2(2)=0.655, p=0.72)$. The pain intensity ratings on the 20 acute electrical stimuli did not differ between the groups $(\chi 2(2)=2.538, p=0.28)$ with median pain ratings in the healthy group of 3 (34.25 ), in the LBLP group of 4.5 (3-6) and in the FBSS group of $4.5(3-6)$ out of 10 (table 2).

\subsection{DCM analysis}

\subsubsection{Model fitting}

\subsubsection{Healthy participants}

The exceedance probability $(x p)$ for families $F(x p=0.371)$ and $L(x p=0.603)$ was far greater than that of the other families (xp varying from 0.0001 towards 0.019) (figure 1). Next, exceedance probabilities of all 114 models were separately evaluated. There was one clear winning model, derived from family $F$, that outperformed the other models with an xp of 0.481 namely model 40 . This model had input from S1 with forward connection towards the left and right ACC and the left and right insula. Additionally, forward connections were found from the left ACC towards the right ACC, from the left insula towards the right insula and from the left PFC towards the right PFC. Left and right insula had forward connections towards respectively the left and right ACC. The left and right ACC on their turn, had forward connections towards respectively the left and right PFC. All forward connections demonstrated a corresponding backward connection. The Bayesian omnibus risk test of this analysis indicated that the null hypothesis of equal evidence for all models could not be rejected (Bayesian omnibus risk $=0.058$ ). 


\subsubsection{Patients with LBLP}

The $x p$ for family $F(x p=0.974)$ outperformed the other families (xp varying from 0.0001 towards 0.007 ) (figure 1). Secondly, exceedance probabilities of all 114 models were separately evaluated. Model 38 (from family F) outperformed the other models with an $x p$ of 0.657 . This model has his input from S1 with forward connection towards the left and right ACC, the left and right insula and the left and right PFC. Additionally, forward connections are found from the left ACC towards the right ACC, from the left insula towards the right insula and from the left PFC towards the right PFC. Left and right insula have forward connections towards respectively the left and right ACC. The left and right ACC on their turn, have forward connections towards respectively the left and right PFC. All forward connections have a corresponding backward connection. The Bayesian omnibus risk test of this analysis indicated that the null hypothesis of equal evidence for all models could be rejected (Bayesian omnibus risk = $0.013)$.

\subsubsection{Patients with FBSS}

Family $F$ had an $x p$ of 0.921 , which was higher than the $x p$ of the other families (xp varying from 0.0001 towards 0.055) (figure 1). Regarding model inference, model 38 had a higher $x p(x p=0.275)$ than the other models. The Bayesian omnibus risk test of this analysis indicated that the null hypothesis of equal evidence for all models could not be rejected (Bayesian omnibus risk $=0.05$ ).

\subsubsection{Parameter estimates}

To determine effective connectivity of parameters, a PEB was performed on the nested models of family F. Estimated connectivity of the parameters that contribute to the model evidence, common across all populations, are represented in figure 2. All posterior probabilities are above 0.75 . Contributing forward and backward connections are all excitatory. The first regressor was used to determine differences between being a patient or not being patient which resulted in differences in the connection from S1 towards right insula (Pp:0.81, scaling parameter: 0.954) and from left PFC towards right PFC (Pp:0.88, scaling parameter: 0.917) (figure 3). Between the two patient groups, differences in the connection from S1 to right ACC (Pp: 0.98, scaling parameter: 0.896), from right INS towards right ACC (Pp: 0.83, scaling parameter: 0.931), from right ACC towards right PFC (Pp: 0.84, scaling parameter: 1.095) and from left PFC to SI (Pp: 0.8, scaling parameter: 1.084). Parameters associated with covariate age are the connection from left ACC towards right ACC (Pp:0.89, scaling parameter: 1.005), from right ACC towards right INS (Pp:0.99, scaling parameter: 0.992) and from left INS towards right INS (Pp:0.87, scaling parameter: 0.995).

\section{Discussion}

In this study, we calculated DCM within a Bayesian framework to determine whether the coupling between brain regions was different in healthy participants, patients with nociceptive LBLP and patients with neuropathic FBSS, when providing painful electrical stimulation on the lower limb. Model families that showed the best explanation for the recorded EEG data all had forward and backward connections. Forward connections are known to segregate sensory information while backward connections are denoted to have a role in mediating contextual effects (Penny et al., 2011). It is already 
demonstrated that pain processing is modulated by social context, attention and certain personality traits of a person (van Heck et al., 2017), which makes it very plausible that forward as well as backward connections are playing a crucial role in nociceptive processing, in both healthy participants and patient groups.

The winning model that is best explaining the processing of an acute nociceptive stimulus in healthy participants, is different from that seen in patient groups. Differences in brain connectivity between healthy controls and pain patients are in line with the abnormal functional connectivity within the default mode network that was found in chronic low back pain patients compared to healthy controls (Tu et al., 2019). Additionally, in 2019 two coordinate-based meta-analyses were performed to reveal spatial patterns of functional activation in response to experimental pain in healthy participants and chronic low back pain patients. In healthy participants, consistent activation in the right anterior hippocampus, parahippocampal gyrus, and amygdala was found. In chronic pain patients, consistently less activation in patients' anterior hippocampus was elucidated compared with healthy participants (Ayoub et al., 2019). In a systematic review of MRI and fMRI studies exploring structural and functional brain changes in chronic low back pain patients, it was found that only 3 of 10 functional MRI studies observed significant differences during noxious stimulation between pain patients and control groups whereas 13 of 16 studies observed significant brain activation differences between pain patients and controls during various external tasks. The authors concluded that brain changes in chronic low back pain patients were mainly observed in areas important in emotion and cognition, rather than those typically associated with nociception (Ng et al., 2018). As such, the occurrence of different winning models in pain patients compared to healthy participants in the currently performed study is in line with available literature.

Between both patient groups however, no differences could be withheld, meaning that, we found no evidence of altered processing of an acute painful stimulus in patients with neuropathic and nonneuropathic LBP. This suggests that despite different clinical manifestations between patients with LBLP and FBSS, the presence of pain is causing similar brain responses. Additionally, presence of the same winning model in both patient groups could be supported by similar levels of increased pain catastrophizing in those patients, compared to healthy participants. Pain catastrophizing is significantly associated with increased activity in several brain areas including regions related to attention to pain, with a pivotal role for the dIPFC, as revealed in patients with fibromyalgia (Gracely et al., 2004). The additional component of the winning model in both patient groups, compared with healthy participants, is the forward and backward connection between S1 and right dIPFC. Previous studies already demonstrated an increased function of the dIPFC during chronic pain states (Seminowicz and Moayedi, 2017). Because its size is enlarged in humans, when compared to other primates, it is thought that the dIPFC is involved in complex cognitive processes (Nee and D'Esposito, 2016; Rahnev et al., 2016). Additionally, in the determination of the strength of parameter estimates, there was a difference in the strength of that particular connection (left dIPFC to S1) between patients with LBLP and FBSS. This leads to the hypothesis that patients could be distinguished from healthy participants due to the absence of a direct connection between S1 and dIPFC in healthy participants. Moreover, one could assume that differences in the strength of this connection can further discriminate between patients with LBLP and FBSS. However, this is only a hypothesis generated through the present study findings and should be further explored. Future studies could also further evaluate the role of pain catastrophizing by including this concept as a covariate in the DCM model building. 
The thalamic nuclei are involved in the sensory and affective components of pain and modulate ascending nociceptive information (Ab Aziz and Ahmad, 2006). Based on literature that cortical evoked potentials are a result of a summation of electrical discharges at different depths and the decreased source-reconstructed signal-to-noise ratio with more in depth sources, we fitted the thalamus as a hidden source (Goldenholz et al., 2009). Therefore, it is possible that the hidden source is representing other midbrain sources than the thalamus, which are contributing to stimulus processing (Boly et al., 2012). This may explain the winning model without thalamus in our study, where this hidden thalamic source is rather serving as a minor key player in contrast to the cortical end target regions.

Despite the differences in brain connectivity, the pain intensity rating of acute nociceptive stimuli was not higher in pain patients compared to healthy participants. Additionally, the intensity at which patients and healthy participants experienced a stimulus as painful, did not differ either. Regarding sensory processing, frequently observed characteristics in patients with chronic pain are allodynia and hyperalgesia (Arendt-Nielsen et al., 2018; Wilder-Smith, 2011). However, the current finding is in line with the findings of Diers et al. (2007) who demonstrated that pain thresholds after intramuscular and intracutaneous electrical stimulation of the M. Erector Spinae and M. Extensor Digitorum were not altered between healthy participants and patients with chronic LBP (Diers et al., 2007).

The methodology of estimating effective connectivity relies on the concepts of DCM and Bayesian principles. A possible drawback of this procedure is that Bayesian model selection is making a relative statement about the goodness of fit of the model, conditional on the model space of the current dataset, meaning the results are always conditional on the models that were constructed (Chen et al., 2008). Therefore, we carefully constructed models, embedded in a family structure to obtain a realistic, well-structured set of models, although it is possible that another model that was not included in this model space, would perform even better. Another concern could be that more complex models will always be favored compared to simpler models, because they can model more features with an excellent fit. In DCM however, parameters of the posterior densities are estimated in a way which maximizes the approximate model evidence. Hence model evidence can be cast into model fit and model complexity, causing a penalization for more complex models (Friston et al., 2007). In other words, DCMs are estimated in a way which optimizes the trade-off between accuracy and complexity and therefore over-fitting is avoided with DCM (Stephan et al., 2010).

In this study we focused on the processing of an acute nociceptive stimulus, however future studies could evaluate resting state processing to determine whether there are differences between healthy participants and chronic LBP patients with different underlying pain mechanisms in resting situations and daily living situations. This study has a couple of limitations such as the use of non-invasive electrodes, leading to an identification of neuronal dynamics by indirect observations on scalp electrodes. A second limitation is that the patients were older than the healthy participants. Therefore, we included an additional covariate to account for age differences. A third limitation is that besides the difference in primary pain mechanism (nociceptive versus neuropathic), both patient groups also differed in the amount of previous surgeries (none versus at least 1 previous spine surgery), pain duration (several weeks to months versus years), anatomical abnormality (present versus absent (only scar tissue)) and pain catastrophizing (no clinically relevant degree of catastrophizing versus a clinically relevant degree of catastrophizing) among other factors. Therefore, differences between both patient groups could also be explained by those factors and not only to differences in pain mechanism. A fourth 
limitation is that skin conductivity could induce an influence on pain thresholds. No specific precautions were taken into account to minimize this influence. Finally, only differences in brain connectivity during processing of an acute painful stimulus were explored in this study. The addition of a control situation, i.e. a non-painful stimulus condition, would provide further insights to distinguish between both underlying pain mechanisms. However, the study also has a couple of strengths such as the fair amount of participants and fitted models and the use of state-of-the-art methods for evaluating effective connectivity with EEG, due to the low cost, limited availability of staff and the noninvasiveness of this method.

\section{Conclusion}

When administrating an electrical nociceptive stimulus, effective connectivity measurements revealed an additional connection between S1 and PFC in patients with non-neuropathic LBLP and patients with neuropathic FBSS, compared to healthy participants.

\section{Methods and materials}

\subsection{Participants}

Healthy participants, presurgical patients with nociceptive low back-related leg pain (LBLP) and patients with neuropathic chronic low back and leg pain due to Failed Back Surgery Syndrome (FBSS) were recruited between March 2016 and January 2018. All participants provided written informed consent prior to participation. The study was conducted according to the revised Declaration of Helsinki (1998). The study protocol was approved by the Ethics Committee of the University Hospital Brussels (B.U.N. 143201628585, 143201526926, 143201526924). Baseline data of the B2asic RCT (Clinicaltrials.gov identifier NCT02630732) and EEG Inspiration trial (Clinicaltrials.gov identifier NCT02751216) were used in this study.

Participants were not allowed to take part in the study if they were pregnant or if they were overconsuming alcohol.

Participants were included as healthy, pain-free controls if they were aged between 18 and 65 years, were free of specific pathology (e.g., spine pathologies, infection, rheumatoid arthritis, malignancy, diagnosed diabetes, cardiovascular or pulmonary problems, psychiatric disorders or other conditions) at the time of the study, were not experiencing any acute and/or chronic pain state at the time of the study and were not taking analgesics or medication for any chronic disease. Exclusion criteria were evidence of specific pathology and currently receiving treatment or medication.

Patients with nociceptive LBLP (Ekedahl et al., 2016; Harrisson et al., 2017; Konstantinou et al., 2018), aged 18-65 years, were recruited at the department of neurosurgery from the University Hospital Brussels. Patients with lumbar radicular nociceptive pain, with an indication for spine surgery, were contacted to participate (Adebajo and Fabule, 2012). All patients had a score $<4 / 10$ on the 'Douleur Neuropathique en 4 Questions (Neuropathic pain in 4 questions)' (DN4). Exclusion criteria were symptoms of spinal cord compression, patients with chronic illness accompanied by uncontrolled chronic pain and patients with polyneuropathy symptoms, rheumatoid, neurological or psychiatric disorders. 
Patients with postsurgical neuropathic pain were recruited from the surgery schedule for neuromodulation, at the department of neurosurgery of the University Hospital Brussels. Patients were recruited if they were above the age of 18 years, diagnosed with FBSS (i.e. the surgical end-stage after one or several operative interventions on the lumbar neuroaxis, indicated to relieve lower back pain, radicular pain or the combination of both, without a positive effect (Follet and Dirks, 1993)) and scheduled for the implantation of a spinal cord stimulator. All patients with FBSS had dominant leg pain (visual analogue scale score $>5 / 10$ ) and chronic back pain, refractory to conservative treatments (pharmacological and physical therapy and minimal invasive pain therapy). Patients were only eligible if they experienced neuropathic leg pain, as screened with the DN4 by a score $\geq 4 / 10$ (Bouhassira et al., 2005), and fulfilled the NeuPSIG criteria (neuroanatomically plausible pain distribution and confirmatory tests (negative or positive sensory signs and/or diagnostic test confirming lesion or disease explaining neuropathic pain)) (Haanpaa et al., 2011).

In the healthy group, 10 males and 10 females were included with a median age of 24 (22-43.5) years. In the group with LBLP patients, 9 males were included and 11 females with a median age of 48.5 (36.75-59.5) years. In the group with FBSS patients, 6 males and 14 females were included with a median age of 52 (49-56) years. There is a significant age difference between the groups $(\times 2(2)=$ $16.755, p<0.001)$, with healthy participants being younger than the patients, but no significant sex difference $(\chi 2(2)=1.7829, p=0.41)$. The PCS score was significantly lower in healthy participants than in both patient groups $(x 2(2)=22.97, p<0.001)$. Between both patient groups, we could not demonstrate a difference in PCS score $(\mathrm{W}=105.5, \mathrm{p}=0.051)$.

\section{$\underline{5.2 \text { Study protocol }}$}

This cross-sectional study consisted of one study visit at the University Hospital Brussels. During the study visit, electrical detection thresholds (EDT) and electrical pain thresholds (EPT) were consecutively determined at the right sural nerve in all participants. Afterwards, participants received 20 electrical stimuli at an intensity of 1.4 times the EPT at the right sural nerve, with simultaneous electroencephalographic (EEG) recordings. Patients received no instructions about medication use and continued to take their current medication. At the beginning of the visit, participants were asked to complete the Pain Catastrophizing Scale (PCS).

\section{$\underline{5.3 \text { Outcome measurements }}$}

Electrical stimuli (constant current pulse train of 5 pulses at $250 \mathrm{~Hz}$ ) were applied on the right sural nerve with a bipolar felt pad electrode which was located posterior of the lateral malleolus (France et al., 2002; Jurth et al., 2014) (Surpass LT Stimulator (EMS Biomedical, Korneuburg, Austria)). Stimuli were gradually increased by steps of $0.5 \mathrm{~mA}$, starting from $0 \mathrm{~mA}$, until the participant experienced a faint sensation (EDT). Stimuli were further increased until the participant experienced an unpleasant sensation (EPT) (Vuilleumier et al., 2015). Measurements were performed three times with 30 seconds interval. The mean of the three measurements was used in all analyses (Vuilleumier et al., 2013). Electrical pain thresholds are a reliable assessment tool for evaluating the sensitivity of the spinal nociceptive pathways in patients with chronic pain (Biurrun Manresa et al., 2011). All participants received 20 electrical stimuli (pulse train of 5 pulses), with an inter-stimulus interval of 8-12 seconds, 
at an intensity of 1.4 times the individualized EPT, to ensure that stimuli were perceived as painful. During the application of the 20 stimuli, EEG recordings were made. After the 20 stimuli, patients were asked to give a pain intensity rating on a verbal numeric rating scale from 0 (no pain) to 10 (worst pain imaginable).

\subsubsection{EEG}

All assessments were conducted in the afternoon between 2 and 8 PM in a quiet, lighted room with the same equipment. Participants were instructed to sit as relaxed and still as possible. Additionally, they were asked to close their eyes, avoid blinking and avoid making small movements with their face. A scalp EEG (Sienna digital EEG, EMS Biomedical, Korneuburg, Austria) with a 32-channel Sn surface electrodes headcap was used (Headcap, EMS Biomedical, Korneuburg, Austria), following the standard 10/20 montage system. Two reference electrodes were located behind the ears (earlobe references). Impedances were evaluated and kept as low as possible. The EEG was sampled against the AFz ground electrode.

Raw data files were exported as text files and imported into EEGLAB (Delorme and Makeig, 2004), a free toolbox in Matlab (MathWorks, Sherbon, MA, USA) for preprocessing of the data following Makoto's preprocessing pipeline (https://sccn.ucsd.edu/wiki/Makoto's_preprocessing_pipeline). The following steps were consecutively executed in EEGLAB to preprocess the data:

- Triggering input was read in through an additional channel and removed afterwards.

- The data was downsampled from $2048 \mathrm{~Hz}$ towards $256 \mathrm{~Hz}$ to compress data size.

- A high-pass filter at $1 \mathrm{~Hz}$ and a low-pass filter at $50 \mathrm{~Hz}$ were applied.

- Channel coordinates were allocated to all electrodes.

- Bad channels were removed with the clean_rawdata function in EEGLAB and interpolated.

- Re-referencing against the average of 32 recorded surface electrodes.

- Independent Component Analysis (ICA) with AMICA.

- Epochs from 1 second pre-stimulus to 2 seconds post-stimulus were created.

- Baseline correction from $-1000 \mathrm{~ms}$ to $0 \mathrm{~ms}$ was executed to compensate for signal drifts.

After the preprocessing, data files were loaded into SPM 12 (Wellcome Trust Centre for Neuroimaging, London, UK), a toolbox in Matlab 2016b. After loading the data into SPM, the first step was to manually check the EEG cap configuration to be sure the electrodes were located at the correct position. Afterwards, the 20 stimuli per participant were averaged to obtain one averaged event related potential (ERP) for each person.

\subsubsection{DCM}

DCMs are generative models of the brain responses, which provide posterior parameter estimates including information about the strength of connections between neuronal populations and the context-dependent modulation (Stephan et al., 2010). DCM for EEG is relying on two models: one realistic neurobiological model about the interacting neural populations and one biophysically plausible forward model for the transformation between the real underlying neural activity and the measured/observed activity (Stephan et al., 2007). Here, the neural state equation describing the evolution of the hidden states is based on the Jansen and Rit model (Jansen and Rit, 1995). A small number of state variables is used to represent brain regions (David et al., 2006). The observation model is a forward model which describes how an electromagnetic field is propagated through the brain and gives rise to electrical potentials at the scalp (David et al., 2006). All parameters of the model are 
estimated using a variational Bayesian estimation scheme (Friston et al., 2007). In general, DCM has state equations of the following form:

$$
\frac{d x}{d t}=f(x, u, \theta)
$$

with $\mathrm{x}$ representing the neuronal state of cortical areas and $\mathrm{u}$ the experimental input. The observer equation is described as a linear forward model of the form:

$$
y=L(\theta) x_{0}+\varepsilon
$$

with y the measured response, $L(\theta)$ the lead field matrix for coupling electrical sources to EEG channels, $\mathrm{x}_{0}$ the transmembrane potential of pyramidal cells and $\varepsilon$ the observational error. (David et al., 2006; Stephan et al., 2007)

\subsubsection{DCM model building}

For the model building, a pre-defined number of brain regions was chosen, based on the existing literature on pain processing of nociceptive stimuli. The included brain regions for DCM consisted of the thalamus (Boly et al., 2012), left primary somatosensory cortex (S1) (Yang et al., 2017), left and right dorsal anterior cingulate cortex (dACC) (Sevel et al., 2015), left and right dorsolateral prefrontal cortex (dIPFC) (Sevel et al., 2015) and left and right posterior insula (Sevel et al., 2015) (table 1). S1 was restricted to the left hemisphere because all participants were stimulated on the right side. All sources are fitted as single equivalent current dipoles. The thalamus is serving as a hidden source due to its deep location within the brain (Friston et al., 2013). Nociceptive information was assumed to act as an experimental input to the thalamus or to $\mathrm{S} 1$ if the thalamus was not included in the model.

Based on current understanding of neurophysiology, we specified 114 connectivity models (appointed with numbers) with different extrinsic connections. Models can be categorized based on the family they belong to, into twelve families (appointed with letters). Model families A, C, E, G, I and K only have forward connections, the other model families have forward and backward connections. Due to the hierarchical structure of the brain, the thalamus is the brain region which is sending forward connections to other brain regions. Connections from other structures back to the thalamus are defined as backward connections. In model families $A$ to $F$, the somatosensory cortex is serving as input structure, while in families $G$ to $L$ the thalamus has this role. Families $A$ and $B$ are consisting of models without the thalamus and are restricted to the left hemisphere. Families $C$ and $D$ entail models without the thalamus with bilateral brain regions. Families $\mathrm{E}$ and $\mathrm{F}$ are similar to $\mathrm{C}$ and $\mathrm{D}$ with the addition of connections between the bilateral brain regions. The construction of families $G$ to $L$ is similar to the families $A$ to $F$, although the thalamus has been added to the model and is receiving the input instead of S1.

All analyses were performed on a time period from stimulus onset (at time 0 ) to $500 \mathrm{~ms}$ post-stimulus. Early responses were not modeled, so modeling was started around the first large deflection with the onset parameter at $64 \mathrm{~ms}$. This is assumed to be a reasonable time delay after which one can expect activation of the cortical area provoked by the stimulus, due to the propagation of the stimulus through the nodes (Litvak et al., 2011). The construction and estimation of the 114 models was performed on each individual participant, resulting in 2280 model fittings ( 20 subjects $x 114$ models) in each group.

\subsubsection{Pain catastrophizing}

The PCS questionnaire was used to measure the level of pain catastrophizing. This questionnaire consists of 13 pain-related cognition items that needed to be scored on a 5 -point Likert scale $(0=$ not 
at all, $4=$ all the time)(Sullivan et al., 1995). Scores $\geq 30 / 52$ indicate a clinically relevant level of catastrophizing (Sullivan, 1995). The internal consistency, test-retest reliability and validity are acceptable (Lame et al., 2008; Osman et al., 1997).

\section{$\underline{5.4 \text { Data analysis }}$}

All analyses were performed with Matlab 2016b (MathWorks, Sherbon, MA, USA) and R studio version 0.99 .903 ( $R$ version 3.4). Shapiro and Levene's tests were used to determine whether parametric or non-parametric statistics were the most appropriate. Demographics were compared between the three groups with a Kruskal-Wallis test for age and a Chi-square test for sex. Pain thresholds and pain intensity ratings between the three groups were compared with Kruskal-Wallis tests.

DCM model fitting was performed in two stages. During the first stage, model inference was conducted independently in the three groups, based on the same set of alternative models to obtain a winning family and winning model in each group. Within the setting of a family comparison, all models within a family are averaged and the exceedance probability, i.e. the probability of one model being more likely than any other model, is calculated for all families (Penny et al., 2011; Stephan et al., 2009). Random-effects Bayesian model comparison (RFX) was applied in this study, due to the robustness and because we assume the optimal model is not identical across subjects (Kasess et al., 2010). Additionally, the Bayesian omnibus risk was calculated to evaluate whether there was a real difference between the models. The Bayesian omnibus risk represents the posterior probability that the model frequencies of all models being tested are equally likely to represent the observed data (Rigoux et al., 2014). During the second stage, Parametric Empirical Bayes (PEB) was used to evaluate group effects on parameters with default settings (Friston et al., 2016). This was performed on the nested models belonging to the family of the winning model. To model the average connectivity over subjects, we included a first regressor. Two additional regressors were added to model between group differences; one regressor for evaluating the differences between healthy participants versus patients and one regressor for evaluating differences between patients. At last, a covariate with mean-centered age for each participant was included to account for age differences. Additionally, parameters that were not contributing to the model evidence, were removed with a search algorithm (Friston and Penny, 2011).

\section{Acknowledgments}

This study was partly funded by the Applied Biomedical Research Program of the Agency for Innovation by Science and Technology, Belgium (IWT-TBM project No. 150180). Eva Huysmans is a predoctoral research fellow of the Research Foundation - Flanders (FWO). Kelly Ickmans is a postdoctoral research fellow of the Research Foundation - Flanders (FWO). Maarten Moens has received speaker fees from Medtonic and Nevro. There are no other conflict of interests to declare. 


\section{References}

Ab Aziz, C.B., Ahmad, A.H., 2006. The role of the thalamus in modulating pain. Malays J Med Sci. 13, 11-8.

Adebajo, A., Fabule, J., 2012. Management of radicular pain in rheumatic disease: insight for the physician. Ther Adv Musculoskelet Dis. 4, 137-47.

Amorim, A.B., Pappas, E., Simic, M., Ferreira, M.L., Tiedemann, A., Jennings, M., Ferreira, P.H., 2016. Integrating Mobile health and Physical Activity to reduce the burden of Chronic low back pain Trial (IMPACT): a pilot trial protocol. BMC Musculoskelet Disord. 17, 36.

Apkarian, A.V., 2015. The brain adapting with pain: contribution of neuroimaging technology to pain mechanisms. Vol. First edition, IASP Press.

Arendt-Nielsen, L., Morlion, B., Perrot, S., Dahan, A., Dickenson, A., Kress, H.G., Wells, C., Bouhassira, D., Mohr Drewes, A., 2018. Assessment and manifestation of central sensitisation across different chronic pain conditions. Eur J Pain. 22, 216-241.

Ayoub, L.J., Barnett, A., Leboucher, A., Golosky, M., McAndrews, M.P., Seminowicz, D.A., Moayedi, M., 2019. The medial temporal lobe in nociception: a meta-analytic and functional connectivity study. Pain. 160, 1245-1260.

Biurrun Manresa, J.A., Neziri, A.Y., Curatolo, M., Arendt-Nielsen, L., Andersen, O.K., 2011. Test-retest reliability of the nociceptive withdrawal reflex and electrical pain thresholds after single and repeated stimulation in patients with chronic low back pain. European Journal of Applied Physiology. 111, 83-92.

Boly, M., Moran, R., Murphy, M., Boveroux, P., Bruno, M.A., Noirhomme, Q., Ledoux, D., Bonhomme, V., Brichant, J.F., Tononi, G., Laureys, S., Friston, K., 2012. Connectivity changes underlying spectral EEG changes during propofol-induced loss of consciousness. J Neurosci. 32, 7082-90.

Bouhassira, D., Attal, N., Alchaar, H., Boureau, F., Brochet, B., Bruxelle, J., Cunin, G., Fermanian, J., Ginies, P., Grun-Overdyking, A., Jafari-Schluep, H., Lanteri-Minet, M., Laurent, B., Mick, G., Serrie, A., Valade, D., Vicaut, E., 2005. Comparison of pain syndromes associated with nervous or somatic lesions and development of a new neuropathic pain diagnostic questionnaire (DN4). Pain. 114, 29-36.

Burns, L.C., Ritvo, S.E., Ferguson, M.K., Clarke, H., Seltzer, Z., Katz, J., 2015. Pain catastrophizing as a risk factor for chronic pain after total knee arthroplasty: a systematic review. J Pain Res. 8, 21-32.

Chehadi, O., Suchan, B., Konietzny, K., Koster, O., Schmidt-Wilcke, T., Hasenbring, M.I., 2017. Gray matter alteration associated with pain catastrophizing in patients 6 months after lumbar disk surgery: a voxel-based morphometry study. Pain Rep. 2, e617.

Chen, C.C., Kiebel, S.J., Friston, K.J., 2008. Dynamic causal modelling of induced responses. Neuroimage. 41, 1293-312.

Coppieters, I., Meeus, M., Kregel, J., Caeyenberghs, K., De Pauw, R., Goubert, D., Cagnie, B., 2016. Relations Between Brain Alterations and Clinical Pain Measures in Chronic Musculoskeletal Pain: A Systematic Review. J Pain. 17, 949-62.

Craner, J.R., Sperry, J.A., Evans, M.M., 2016. The Relationship Between Pain Catastrophizing and Outcomes of a 3-Week Comprehensive Pain Rehabilitation Program. Pain Med. 17, 20262035.

David, O., Kiebel, S.J., Harrison, L.M., Mattout, J., Kilner, J.M., Friston, K.J., 2006. Dynamic causal modeling of evoked responses in EEG and MEG. Neuroimage. 30, 1255-72. 
Delorme, A., Makeig, S., 2004. EEGLAB: an open source toolbox for analysis of single-trial EEG dynamics including independent component analysis. J. Neurosci. Methods. 134, 9-21.

Diers, M., Koeppe, C., Diesch, E., Stolle, A.M., Holzl, R., Schiltenwolf, M., van Ackern, K., Flor, H., 2007. Central processing of acute muscle pain in chronic low back pain patients: an EEG mapping study. J Clin Neurophysiol. 24, 76-83.

Edwards, R.R., Dworkin, R.H., Sullivan, M.D., Turk, D.C., Wasan, A.D., 2016. The Role of Psychosocial Processes in the Development and Maintenance of Chronic Pain. J Pain. 17, T70-92.

Ekedahl, H., Jonsson, B., Annertz, M., Frobell, R.B., 2016. Three week results of transforaminal epidural steroid injection in patients with chronic unilateral low back related leg pain: The relation to MRI findings and clinical features. J Back Musculoskelet Rehabil. 29, 693-702.

Follet, K., Dirks, B., 1993. Etiology and evaluation of the failed back surgery syndrome. Neurosurg Q. 3, 40-59.

France, C.R., France, J.L., al'Absi, M., Ring, C., McIntyre, D., 2002. Catastrophizing is related to pain ratings, but not nociceptive flexion reflex threshold. Pain. 99, 459-63.

Friston, K., Mattout, J., Trujillo-Barreto, N., Ashburner, J., Penny, W., 2007. Variational free energy and the Laplace approximation. Neuroimage. 34, 220-34.

Friston, K., Penny, W., 2011. Post hoc Bayesian model selection. Neuroimage. 56, 2089-99.

Friston, K., Moran, R., Seth, A.K., 2013. Analysing connectivity with Granger causality and dynamic causal modelling. Curr Opin Neurobiol. 23, 172-8.

Friston, K.J., 1994. Functional and effective connectivity in neuroimaging: a synthesis. Human Brain Mapping. 2, 56-78.

Friston, K.J., Litvak, V., Oswal, A., Razi, A., Stephan, K.E., van Wijk, B.C.M., Ziegler, G., Zeidman, P., 2016. Bayesian model reduction and empirical Bayes for group (DCM) studies. Neuroimage. $128,413-431$.

Goldenholz, D.M., Ahlfors, S.P., Hamalainen, M.S., Sharon, D., Ishitobi, M., Vaina, L.M., Stufflebeam, S.M., 2009. Mapping the signal-to-noise-ratios of cortical sources in magnetoencephalography and electroencephalography. Hum Brain Mapp. 30, 1077-86.

Gracely, R.H., Geisser, M.E., Giesecke, T., Grant, M.A., Petzke, F., Williams, D.A., Clauw, D.J., 2004. Pain catastrophizing and neural responses to pain among persons with fibromyalgia. Brain. $127,835-43$.

Gustin, S.M., Peck, C.C., Wilcox, S.L., Nash, P.G., Murray, G.M., Henderson, L.A., 2011. Different pain, different brain: thalamic anatomy in neuropathic and non-neuropathic chronic pain syndromes. J Neurosci. 31, 5956-64.

Haanpaa, M., Attal, N., Backonja, M., Baron, R., Bennett, M., Bouhassira, D., Cruccu, G., Hansson, P., Haythornthwaite, J.A., lannetti, G.D., Jensen, T.S., Kauppila, T., Nurmikko, T.J., Rice, A.S., Rowbotham, M., Serra, J., Sommer, C., Smith, B.H., Treede, R.D., 2011. NeuPSIG guidelines on neuropathic pain assessment. Pain. 152, 14-27.

Harrisson, S.A., Stynes, S., Dunn, K.M., Foster, N.E., Konstantinou, K., 2017. Neuropathic Pain in Low Back-Related Leg Pain Patients: What Is the Evidence of Prevalence, Characteristics, and Prognosis in Primary Care? A Systematic Review of the Literature. J Pain. 18, 1295-1312.

Hong, J.H., Kim, H.D., Shin, H.H., Huh, B., 2014. Assessment of depression, anxiety, sleep disturbance, and quality of life in patients with chronic low back pain in Korea. Korean J Anesthesiol. 66, 444-50.

Jansen, B.H., Rit, V.G., 1995. Electroencephalogram and visual evoked potential generation in a mathematical model of coupled cortical columns. Biol Cybern. 73, 357-66. 
Jurth, C., Rehberg, B., von Dincklage, F., 2014. Reliability of subjective pain ratings and nociceptive flexion reflex responses as measures of conditioned pain modulation. Pain Res Manag. 19, 93-6.

Kasess, C.H., Stephan, K.E., Weissenbacher, A., Pezawas, L., Moser, E., Windischberger, C., 2010. Multi-subject analyses with dynamic causal modeling. Neuroimage. 49, 3065-74.

Konstantinou, K., Dunn, K.M., Ogollah, R., Lewis, M., van der Windt, D., Hay, E.M., 2018. Prognosis of sciatica and back-related leg pain in primary care: the ATLAS cohort. Spine J. 18, 1030-1040.

Lame, I.E., Peters, M.L., Kessels, A.G., Van Kleef, M., Patijn, J., 2008. Test--retest stability of the Pain Catastrophizing Scale and the Tampa Scale for Kinesiophobia in chronic pain over a longer period of time. J Health Psychol. 13, 820-6.

Litvak, V., Mattout, J., Kiebel, S., Phillips, C., Henson, R., Kilner, J., Barnes, G., Oostenveld, R., Daunizeau, J., Flandin, G., Penny, W., Friston, K., 2011. EEG and MEG data analysis in SPM8. Comput Intell Neurosci. 2011, 852961.

Macfarlane, G.J., 2016. The epidemiology of chronic pain. Pain. 157, 2158-9.

Meints, S.M., Edwards, R.R., 2018. Evaluating psychosocial contributions to chronic pain outcomes. Prog Neuropsychopharmacol Biol Psychiatry.

Meucci, R.D., Fassa, A.G., Faria, N.M., 2015. Prevalence of chronic low back pain: systematic review. Rev Saude Publica. 49.

Nee, D.E., D'Esposito, M., 2016. The hierarchical organization of the lateral prefrontal cortex. Elife. 5.

Ng, S.K., Urquhart, D.M., Fitzgerald, P.B., Cicuttini, F.M., Hussain, S.M., Fitzgibbon, B.M., 2018. The Relationship Between Structural and Functional Brain Changes and Altered Emotion and Cognition in Chronic Low Back Pain Brain Changes: A Systematic Review of MRI and fMRI Studies. Clin J Pain. 34, 237-261.

Osman, A., Barrios, F.X., Kopper, B.A., Hauptmann, W., Jones, J., O'Neill, E., 1997. Factor structure, reliability, and validity of the Pain Catastrophizing Scale. J Behav Med. 20, 589-605.

Penny, W.D., Friston, K.J., Ashburner, J.T., Kiebel, S.J., Nichols, T.E., 2011. Statistical parametric mapping: the analysis of functional brain images. Vol., Elsevier, Amsterdam, The Netherlands.

Rahnev, D., Nee, D.E., Riddle, J., Larson, A.S., D'Esposito, M., 2016. Causal evidence for frontal cortex organization for perceptual decision making. Proc Natl Acad Sci U S A. 113, 6059-64.

Rigoux, L., Stephan, K.E., Friston, K.J., Daunizeau, J., 2014. Bayesian model selection for group studies - revisited. Neuroimage. 84, 971-85.

Seminowicz, D.A., Wideman, T.H., Naso, L., Hatami-Khoroushahi, Z., Fallatah, S., Ware, M.A., Jarzem, P., Bushnell, M.C., Shir, Y., Ouellet, J.A., Stone, L.S., 2011. Effective treatment of chronic low back pain in humans reverses abnormal brain anatomy and function. J Neurosci. 31, 7540-50.

Seminowicz, D.A., Moayedi, M., 2017. The Dorsolateral Prefrontal Cortex in Acute and Chronic Pain. J Pain. 18, 1027-1035.

Sevel, L.S., Craggs, J.G., Price, D.D., Staud, R., Robinson, M.E., 2015. Placebo analgesia enhances descending pain-related effective connectivity: a dynamic causal modeling study of endogenous pain modulation. J Pain. 16, 760-8.

Spahr, N., Hodkinson, D., Jolly, K., Williams, S., Howard, M., Thacker, M., 2017. Distinguishing between nociceptive and neuropathic components in chronic low back pain using behavioural evaluation and sensory examination. Musculoskelet Sci Pract. 27, 40-48.

Stephan, K.E., Harrison, L.M., Kiebel, S.J., David, O., Penny, W.D., Friston, K.J., 2007. Dynamic causal models of neural system dynamics:current state and future extensions. J Biosci. 32, 129-44. 
Stephan, K.E., Penny, W.D., Daunizeau, J., Moran, R.J., Friston, K.J., 2009. Bayesian model selection for group studies. Neuroimage. 46, 1004-17.

Stephan, K.E., Penny, W.D., Moran, R.J., den Ouden, H.E., Daunizeau, J., Friston, K.J., 2010. Ten simple rules for dynamic causal modeling. Neuroimage. 49, 3099-109.

Sullivan, M.J.L., 1995. The Pain Catstrophizing Scale: User Manual. Vol., ed.^eds., pp. 36.

Sullivan, M.J.L., Bishop, S.R., Pivik, J., 1995. The Pain Catastrophizing Scale: development and validation. Psychol Assess. 7, 524-532.

Tu, Y., Jung, M., Gollub, R.L., Napadow, V., Gerber, J., Ortiz, A., Lang, C., Mawla, I., Shen, W., Chan, S.T., Wasan, A.D., Edwards, R.R., Kaptchuk, T.J., Rosen, B., Kong, J., 2019. Abnormal medial prefrontal cortex functional connectivity and its association with clinical symptoms in chronic low back pain. Pain. 160, 1308-1318.

van Heck, C.H., Driessen, J.M.A., Amato, M., van den Berg, M.N., Bhandari, P., Bilbao-Broch, L., Farres-Casals, J., Hendriks, M., Jodzio, A.C., Luque-Ballesteros, L., Schochl, C., VelascoAngeles, L.R., Weijer, R.H.A., van Rijn, C.M., Jongsma, M.L.A., 2017. Pain Processing in a Social Context and the Link with Psychopathic Personality Traits-An Event-Related Potential Study. Front Behav Neurosci. 11, 180.

Vuilleumier, P.H., Besson, M., Desmeules, J., Arendt-Nielsen, L., Curatolo, M., 2013. Evaluation of Anti-Hyperalgesic and Analgesic Effects of Two Benzodiazepines in Human Experimental Pain: A Randomized Placebo-Controlled Study. PLoS One. 8, e43896.

Vuilleumier, P.H., Biurrun Manresa, J.A., Ghamri, Y., Mlekusch, S., Siegenthaler, A., Arendt-Nielsen, L., Curatolo, M., 2015. Reliability of Quantitative Sensory Tests in a Low Back Pain Population. Reg Anesth Pain Med. 40, 665-73.

Webster, L.R., Markman, J., 2014. Medical management of chronic low back pain: efficacy and outcomes. Neuromodulation. 17 Suppl 2, 18-23.

Wilder-Smith, O.H., 2011. Chronic pain and surgery: a review of new insights from sensory testing. J Pain Palliat Care Pharmacother. 25, 146-59.

Yang, Y., Guliyev, B., Schouten, A.C., 2017. Dynamic Causal Modeling of the Cortical Responses to Wrist Perturbations. Front Neurosci. 11, 518. 


\section{Figures}

\section{FAMILIES}

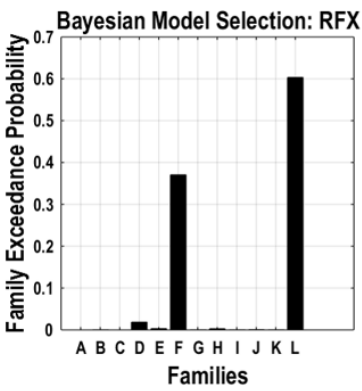

II

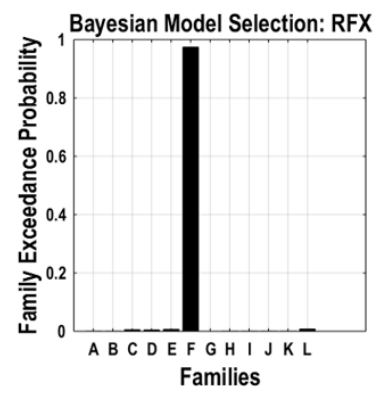

III

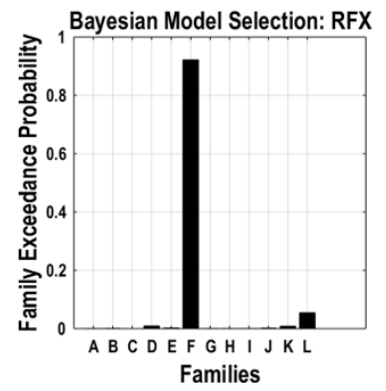

MODELS
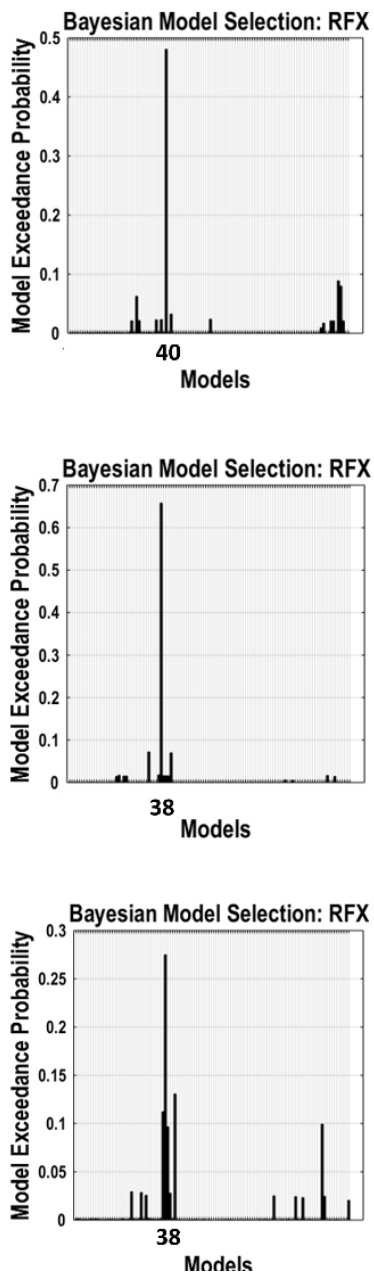

WINNING MODEL
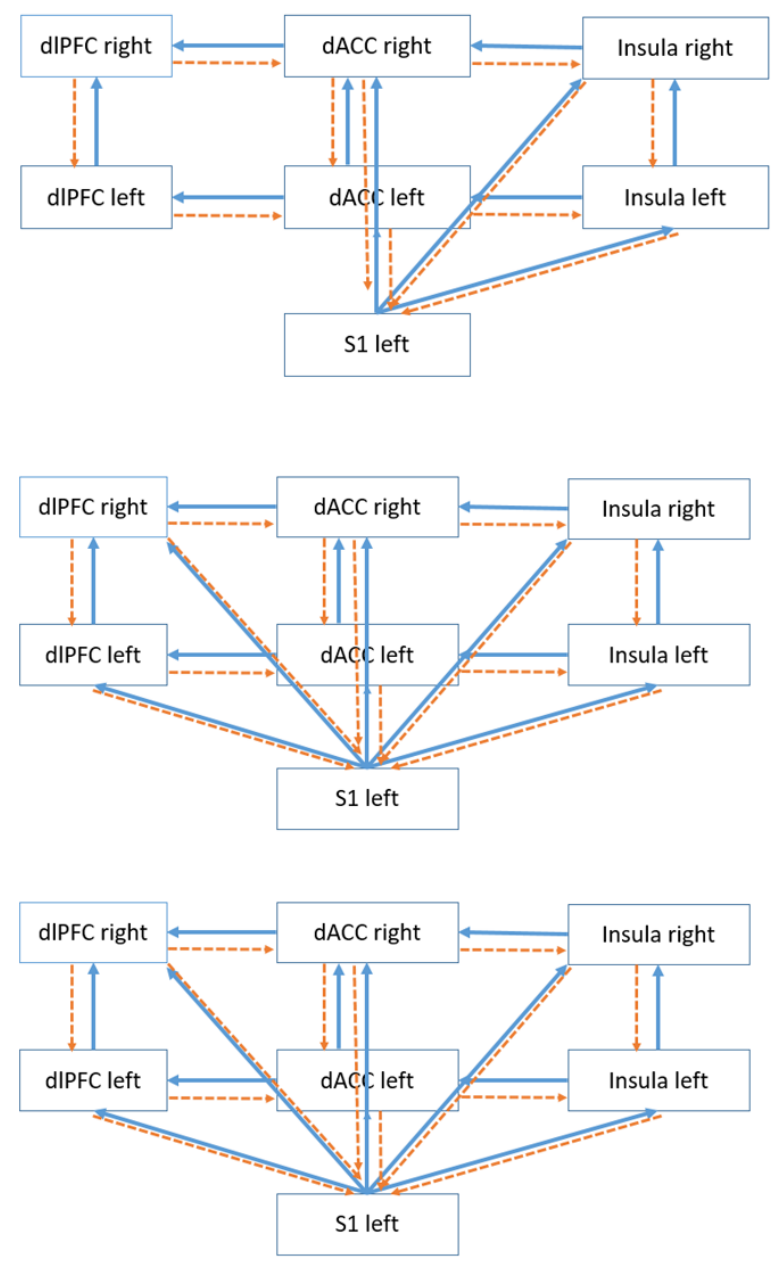

Figure 1: Results of the Bayesian model selection in healthy participants, patients with LBLP and patients with FBSS. In the first column, the exceedance probabilities of the model families are shown. Families $L$ and $F$ outperform the other models in the healthy group (I). Family $F$ outperforms all the other models in the LBLP (II) and FBSS group (III). In the middle column, all models are presented with a clear advantage for model 40 in the healthy group and model 38 in the LBLP and FBSS group. In the right column, the structure of the winning model is represented for each group. Solid blue arrows represent forward connections, dotted orange arrows backward connections between the brain regions. Abbreviations: $\mathrm{dACC}=$ dorsal anterior cingulate cortex, $\mathrm{PFC}=$ dorsolateral prefrontal cortex, S1 $=$ primary somatosensory cortex. 


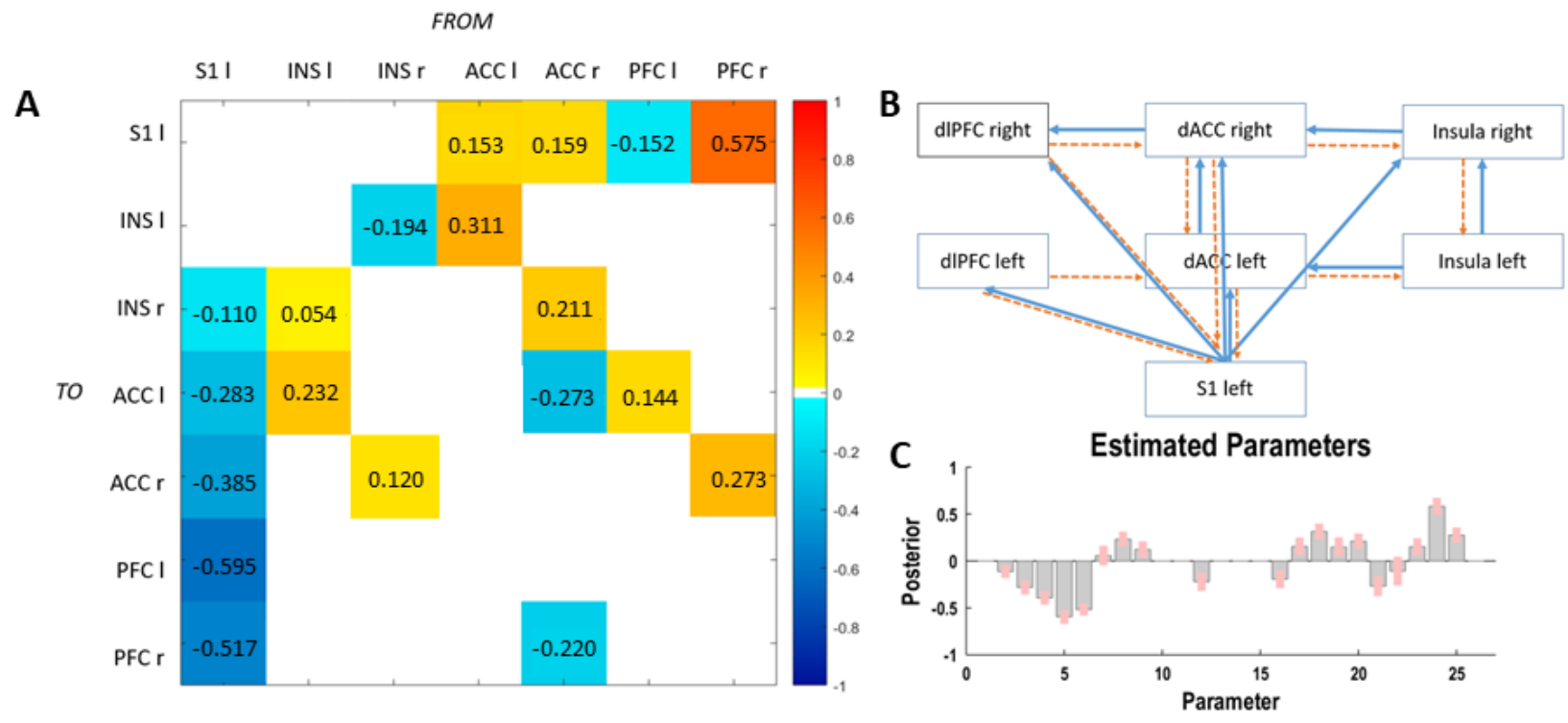

Figure 2: Posterior parameter estimates.

A. Estimated effective connectivity (directed from columns to rows) with parameters that contribute to the model evidence on the log scale. All groups contribute to the mean posterior estimates.

B. The model with all parameters that contribute to the model evidence. Solid blue lines represent forward connections and orange dotted lines backward connections.

C. Posterior estimates of the contributing parameters.

Abbreviations: $\mathrm{ACC}=$ dorsal anterior cingulate cortex, INS = insula, l=left, PFC = dorsolateral prefrontal cortex, $r=$ right, $\mathrm{S} 1$ = primary somatosensory cortex. 

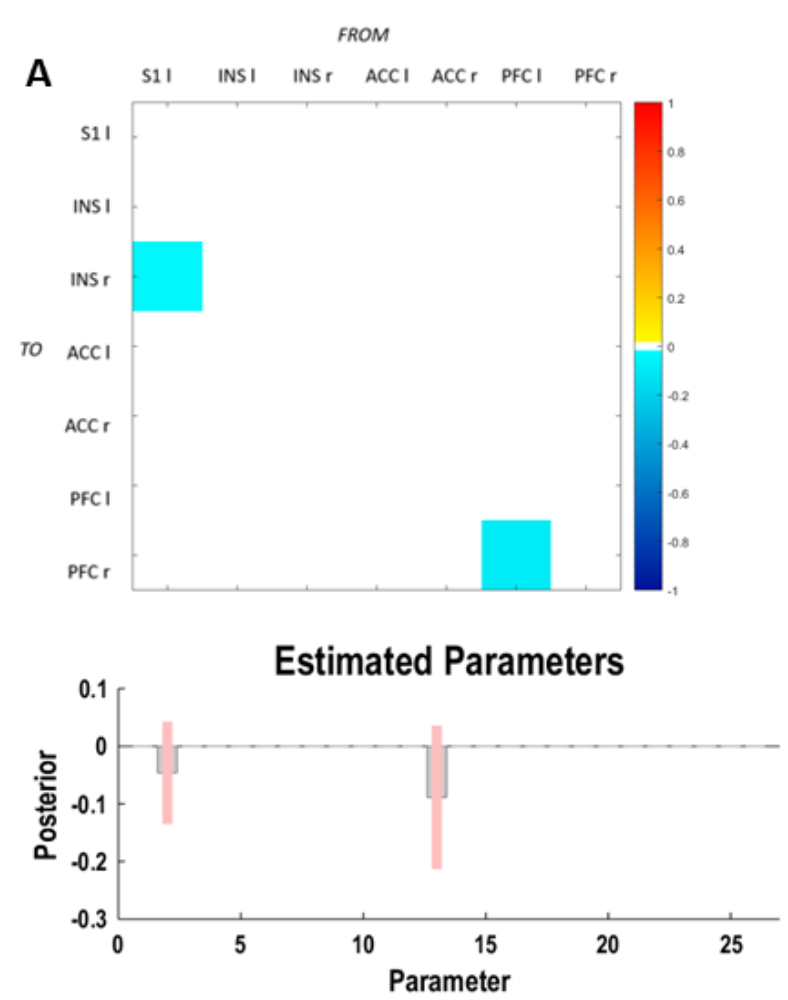

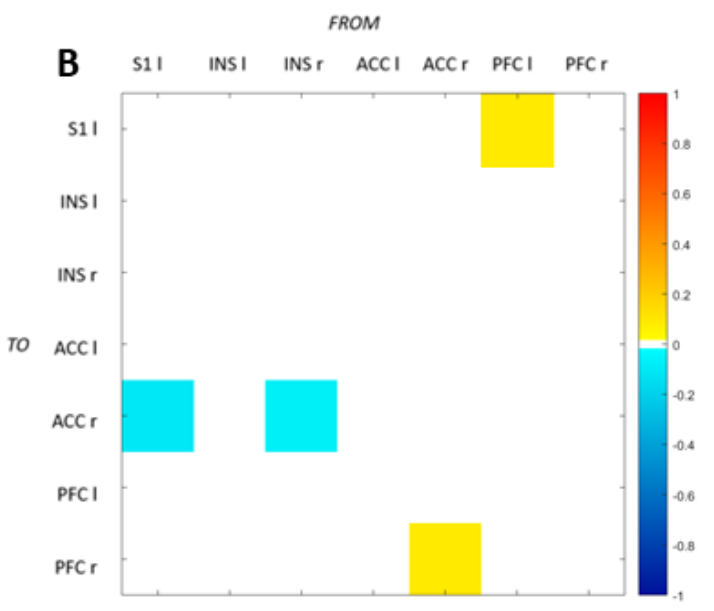

Estimated Parameters

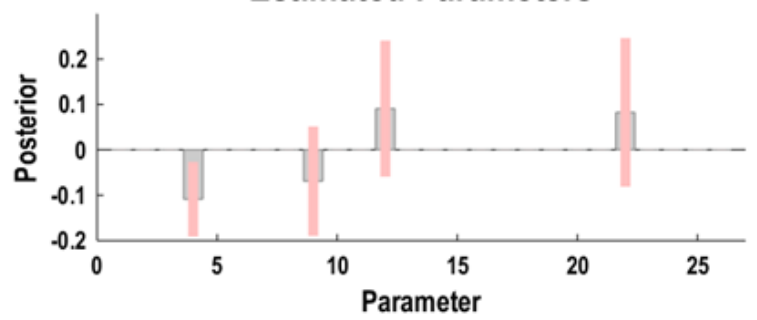

Figure 3: Posterior parameter estimates for comparisons between groups.

A. Contrast between being a patient or not being a patient

B. Contrast between patients with LBLP and patients with FBSS

Abbreviations: $\mathrm{ACC}=$ dorsal anterior cingulate cortex, INS = insula, I=left, PFC = dorsolateral prefrontal cortex, $r=$ right, $\mathrm{S} 1$ = primary somatosensory cortex. 


\section{Tables}

\begin{tabular}{lccc}
\hline Source & $\mathbf{x}$ & $\mathbf{y}$ & $\mathbf{z}$ \\
\hline Thalamus & 500 & 500 & 500 \\
\hline S1 left & -26 & -40 & 68 \\
\hline dACC left & -9 & 8 & 44 \\
\hline PFC left & -39 & 29 & 26 \\
\hline Post insula left & -45 & -25 & 14 \\
\hline dACC right & 9 & 11 & 41 \\
\hline PFC right & 42 & 35 & 26 \\
\hline Post insula right & 45 & -19 & 17 \\
\hline
\end{tabular}

Table 1: MNI coordinates of the included sources in model fitting. Abbreviations: $\mathrm{dACC}=$ dorsal anterior cingulate cortex, $\mathrm{PFC}=$ dorsolateral prefrontal cortex, post= posterior, $\mathrm{S} 1$ = primary somatosensory cortex. 


\begin{tabular}{|c|c|c|c|c|c|}
\hline & $\begin{array}{l}\text { Healthy } \\
\text { participants }\end{array}$ & LBLP patients & FBSS patients & P-value & $\begin{array}{l}\text { Post-hoc } \\
\text { testing }\end{array}$ \\
\hline $\operatorname{Sex}(M / F)$ & $10 / 10$ & $9 / 11$ & $6 / 14$ & 0.4101 & \\
\hline Age (years) & $\begin{array}{l}24(22- \\
43.5)\end{array}$ & $\begin{array}{l}48.5(36.75- \\
59.5)\end{array}$ & $52(49-56)$ & 0.0002 & $\begin{array}{l}\text { HC vs } \\
\text { LBLP: } \\
0.002 \\
\text { HC vs } \\
\text { FBSS: } \\
0.0001\end{array}$ \\
\hline Painful side (L/R) & & $12 / 8$ & $12 / 8$ & 1 & \\
\hline PCS score & $\begin{array}{l}14(8.25- \\
18.25)\end{array}$ & $\begin{array}{l}24.5(17.75- \\
31.5)\end{array}$ & $37(27-43)$ & $<0.0001$ & $\begin{array}{l}\text { HC vs } \\
\text { LBLP: } \\
0.0002 \\
\text { HC vs } \\
\text { FBSS: } \\
<0.0001\end{array}$ \\
\hline EDT (mA) & $\begin{array}{l}1.92(1.46- \\
2.17)\end{array}$ & $2.08(1.50-2.54)$ & $2.33(1.25-3.04)$ & 0.4179 & \\
\hline EPT (mA) & $\begin{array}{l}7.17(4.58- \\
9.08)\end{array}$ & $5.75(4.12-8.42)$ & $5.58(3.83-8.75)$ & 0.7207 & \\
\hline NRS score & $3(3-4.25)$ & $4.5(3-6)$ & $4.5(3-6)$ & 0.2811 & \\
\hline Pain medication & & $\begin{array}{l}\text { Weak opioids: } 1 \\
\text { Weak opioids + } \\
\text { paracetamol: } 3 \\
\text { Weak opioids + } \\
\text { NSAID: } 2 \\
\text { Duloxetine + } \\
\text { paracetamol: } 1 \\
\text { NSAID + } \\
\text { paracetamol: } 5 \\
\text { Paracetamol: } 2 \\
\text { NSAID : } 1 \\
\text { NSAID + } \\
\text { Pregabaline : } 1 \\
\text { None: } 4\end{array}$ & $\begin{array}{l}\text { Amitriptyline: } 1 \\
\text { Gabapentine + weak opioids: } \\
1 \\
\text { Paracetamol: } 3 \\
\text { Pregabaline: } 4 \\
\text { Pregabaline + weak opioids: } \\
4 \\
\text { None: } 1 \\
\text { NSAID: } 2 \\
\text { Opioids: } 1 \\
\text { Duloxetine: } 1 \\
\text { Amitriptyline + pregabaline: } \\
1 \\
\text { Weak opioids: } 1\end{array}$ & & \\
\hline
\end{tabular}

Table 2: Demographics, thresholds and pain intensity ratings of the three groups.

Abbreviations: EDT: electrical detection threshold, EPT: electrical pain threshold, F: female, FBSS:

failed back surgery syndrome, HC: healthy controls, L: left, LBLP: low back-related leg pain, M: male, NRS: numeric rating scale, R:right. 